\title{
SysMon GUI
}

\author{
Snehal Rajesh Lalage ${ }^{1}$,Naveen Kumar Varganti ${ }^{2}$, \\ R.V. Jagannadha Rao Doddi ${ }^{3}$ and Satya Venkata Pramod Kumar Konda ${ }^{4}$ \\ ${ }^{1}$ RV College of Engineering, Bengaluru, India \\ ${ }^{2,3,4}$ Qualcomm Inc., Hyderabad \\ ${ }^{1}$ snehalrajeshl.ec17@rvce.edu.in, 2nvargant@qti.qualcomm.com, \\ ${ }^{3}$ rdoddi@qti.qualcomm.com, ${ }^{4}$ pramodp@qti.qualcomm.com
}

\begin{abstract}
The goal of this project is to make SysMonApp a graphical user interface. SysMonApp is an Android programme that interfaces with Q6s on (Appliation DSP)ADSP/(Computational DSP)CDSP/(Sensor DSP)SDSP subsystems over FastRPC and supports different user functions such as profiling Q6 workload, getting clock information, setting/removing clocks, and getting software thread information, among other things. SysMonApp offers a variety of features, each with its own set of parameters. Users have a hard time remembering many arguments. The user interface will make it easy for users to access all of the functions in one location
\end{abstract}

Keywords: Frontend, backend

\section{INTRODUCTION}

SysMonGUI is a desktop application Graphical User Interface (GUI), which Interacts with the Connected Android Devices and measures the Profiling data. It provides various users functionalities like profiling Q6 workload, get the clock info, set/remove the core and bus clocks, get Software (SW) thread info, get the SW thread level profile stats amongst other supported functionalities. SysMon can be used by all mobile, Internet Of Things (IOT) and automotive operating systems. The application interacts with the Devices using the Android Debug Bridge (ADB) tool and controls the various commands automatically to minimize the User Manual intervention for operating the Device for Profiling purpose. To do different actions, users need to remember different commands, paths etc. The SysMonGUI provides user-friendly User Interface (UI) which monitors different q6's Digital Signal Processor (DSP)s with android Application GUI where user can easily interact with the devices and chipsets for bus profiling and system monitoring. This is a GUI which will display many PMU metrics to understand performance of algorithms, help to debug performance and power issues and to measure chip system modules and bus-profiling of the various chipsets.

The most effective designs for developing a functional GUI lies in the the user-friendly, and aesthetic appeal apart from the functions and features that the app may provide. Another important point that is focused on is that by creating responsive web apps, the GUI is more appealing to use. By carefully selecting the programming languages and the frameworks to be used to design the GUI, it can reduce the number of errors, and will help in making the development process easier, at the same time making the app more light and fast. Using Bootstrap improves the aesthetic appeal of the GUI, and at the same time keeps the app light and efficient. Bootstrap is also very user-friendly and compatible with mobile devices, making it the perfect choice for front-end development.

\section{Design of SysMon GUI}

The overall application has several functionalities and features, each having its own particular use and priority. To make the apps various features easily accessible and user friendly, the 
various features are grouped into six tabs. On clicking these tabs, the user is directed to different pages that have their own specific functionality. These 6 tabs are placed on the top of the screen called the NavBar. Only one tab can be opened at any given point in time. The features and functionalities of each tab is explained in the sections below. The layout of each tab is also mentioned

2.1 Home page:The Home tab is the first tab of SysMonGUI. It is the default tab that opens when the application is launched. In this tab, the user has to select the desired device from the connected devices dropdown. The list of connected devices will populate once the application is launched. The user is supposed to select the device from the list of connected devices.

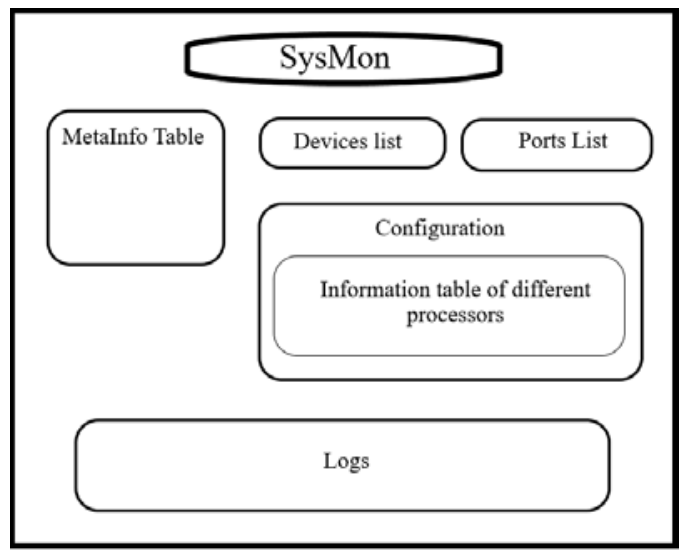

Fig 1: Layout of home page

MetaInfo table and tables of different q6 processors with version and hardware information is displayed. The logs window located at the bottom of the screen displays the current status of the application. The logs are refreshed in real time. The layout of the home tab is shown in Figure 1.

2.2 Profiler page: The second tab of SysMonGUI is the profiler. The profiler tab deals with the metrics of the selected device. In the Home page, the user can click on the processor subsystem and the user will be redirected to the profiler tab with the processor subsystem selected. The user can select the desired clock frequencies and latency. The user can also select which metric that is desired to be evaluated which is present on the left side of the screen. On the bottom half of the screen, the plots showing the performance of the processors with the applied clock frequencies and latency is displayed. Figure 2 shows the layout of the profiler tab.

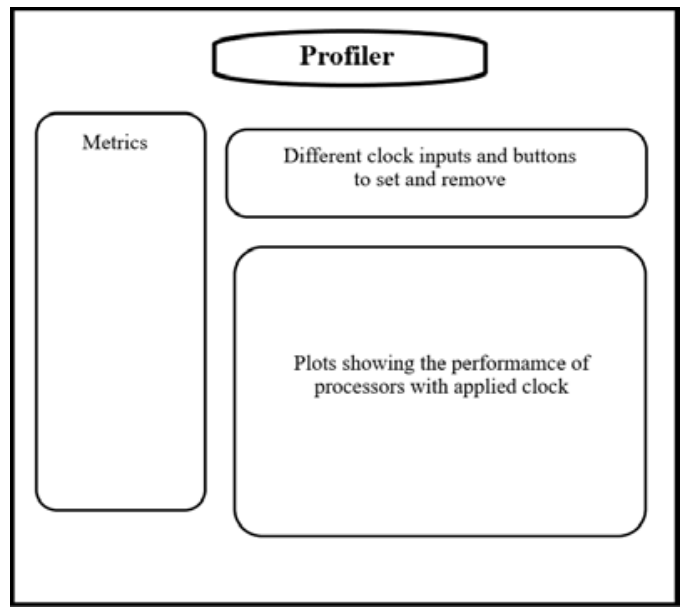

Fig 2: Layout of Profiler page

2.3 Compare page: The third tab of SysMonGUI is the Compare tab. In this tab, the versions of old and upgraded processor will be displayed. Plots between the different versions of the processor will be displayed, based on the selected comparison parameter which is present on the top left of the screen. Graphs of performance of both versions of the same processor is displayed, and the user can gain insights into the performance 
improvements or degrades between different versions of the same processor. Figure 3.3 shows the layout of the compare tab.

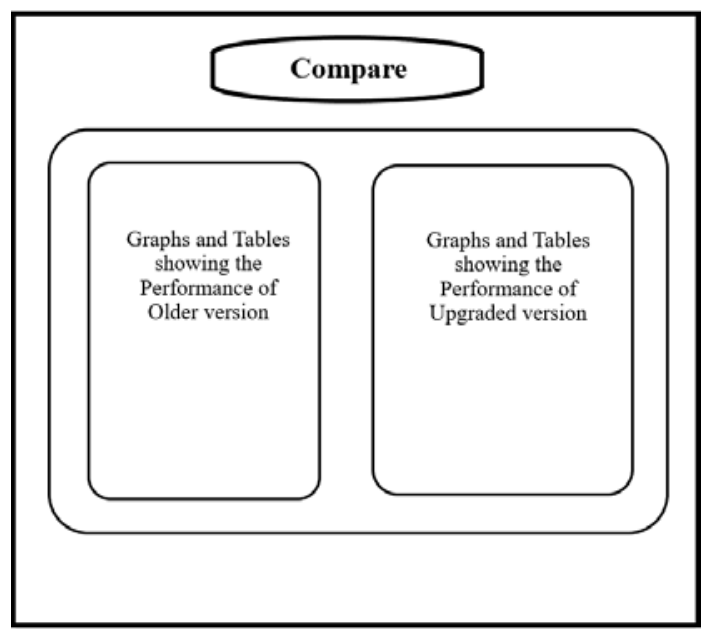

Fig 3: Layout of Compare page

2.4 Services page: The services tab is the fourth tab of SysMonGUI. There are different services provided by SysMonApp like getstate service, clock service and many more that will be explained in detail in Chapter 5. The User can select the service which is present on the left, and the tables and graphs for that particular service will be displayed on the right. The layout of the services tab is shown in Figure 4.

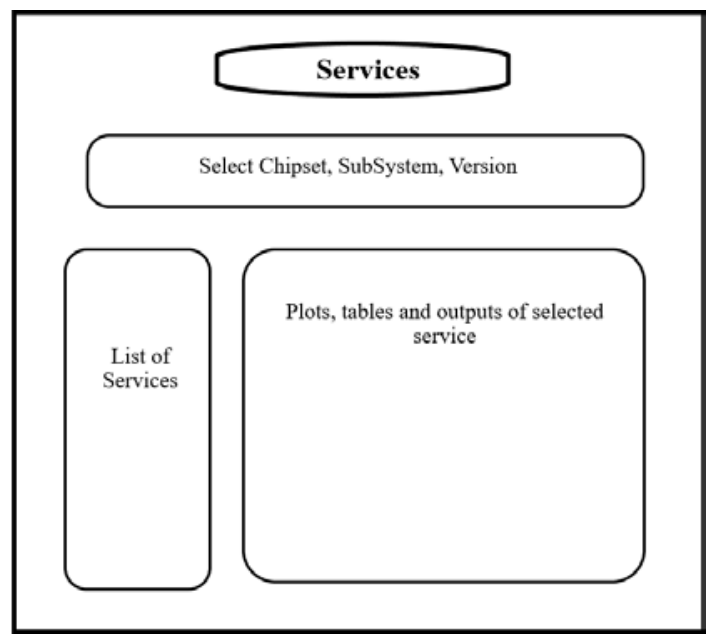

Fig 4: Layout of Services page

2.5 Report page: The reports tab is the fifth tab of the SysMonGUI. This tab is to generate reports about the selected processor. The selected Chipset name, subsystem and version is displayed on the top right of the screen. A detailed report of the performance of the processor is displayed. The layout of the reports tab is shown in Figure 5 


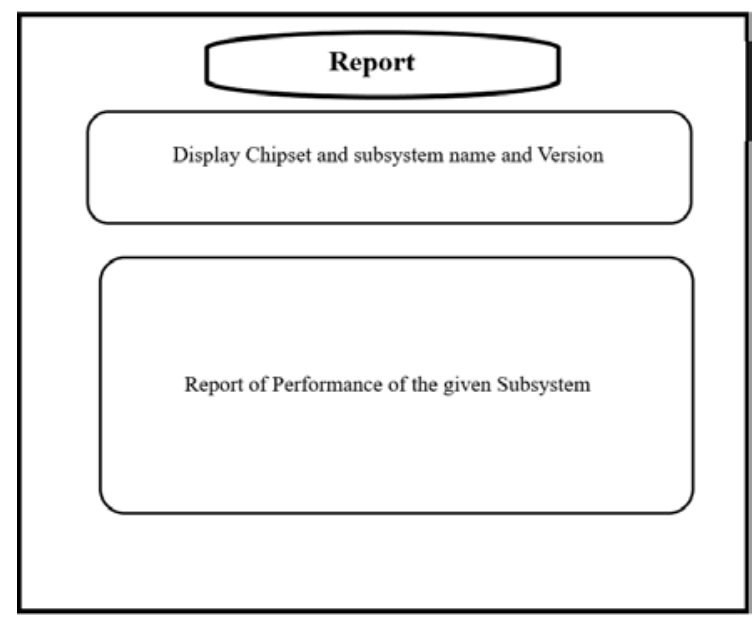

Fig 5 : Layout of Report page

2.6 User Guide page: The sixth and final tab of SysMonGUI is the User Guides tab. In this tab, the instructions about all the tabs and SysMon can be displayed. The user can select the desired option on the left, and instructions and details about that tab appears on the window on the right side of the screen. The layout of the User Guides tab is shown in Figure 6.

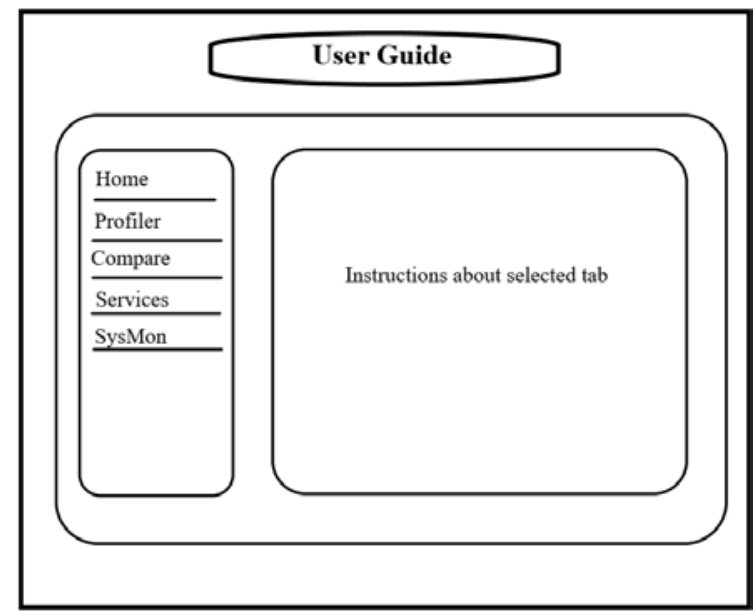

Fig 6: Lay out User Guide page

\section{Implementation of SysMonGUI}

The implementation of SysMonGUI can be split into two parts,

- Frontend - Includes the layout and design aspect of the GUI. This acts as the interface with the user. The frontend is what is displayed, and the user can interact with it directly.

- Backend - The backend is responsible for the inner workings of the application, that is connected to the frontend. The data input from the user (from the frontend), for example, a button click is sent to the backend for processing.

3.1 Home page:Once the UI has been launched, the homepage will appear. To acquire the connected device list, JavaScript in the backend will use exec to run shell commands. It will pop up a window reading "No devices attached!" if there are no devices connected. If devices are connected, a shell command will be run to obtain a list of connected devices, which will then be displayed on the UI. The user must choose a device from a list. The MetaInfo table will show the build information. Every $\mathrm{T}$ seconds, a table displaying the version, frequency, and clock information of the various Q6 processors on the chip will be presented and updated. 


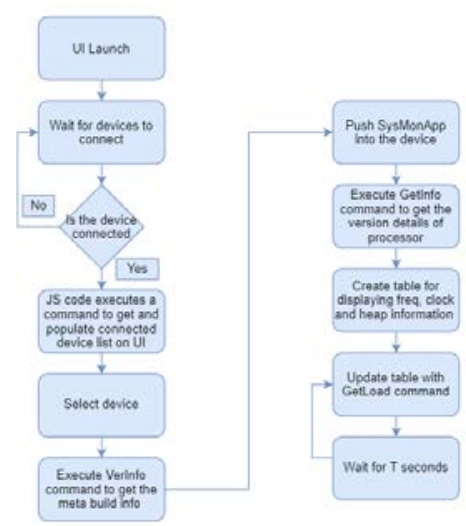

Fig 7: Flowchart of home page

3.2 Profiler page: When the user selects Profiler from the NavBar, the Profiler tab will open. The stats of the selected processor will be provided in a side menu. The user must select a value for coreClock, busClock, and sleep delay before pressing the setClock button. That will set the clocks to the specified numbers, and the backend code will execute and evaluate the processor's performance, which will be displayed as a graph. RemoveClock button will set the clock values back to their default values.

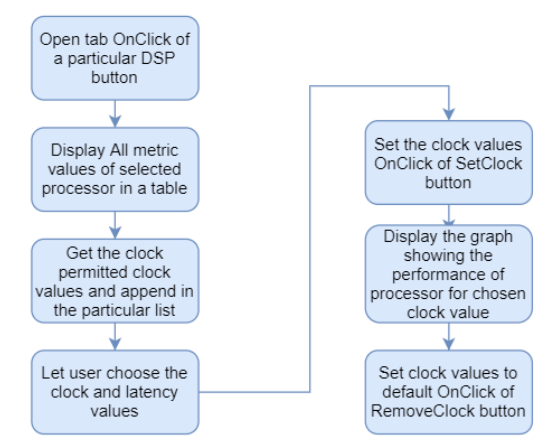

Fig 8: Flowchart of Profiler page

3.3 Compare page: The Compare tab will open when the user selects compare from the NavBar. The name of the chipset, the subsystem, and the versions will be displayed in a table. The user must choose one of the performance details from a list. In the backend, JS code will be executed to obtain the appropriate findings for both versions and present them using graphs.

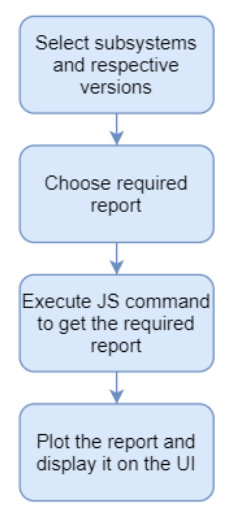

Fig 9: Flowchart of Compare page

3.4 Services page: When the user selects Services from the NavBar, the Services tab will appear. Getstate, Clock services, Tinfo services, and other services are available through SysMonApp. The user must select one of them, and the result will be displayed on the UI 


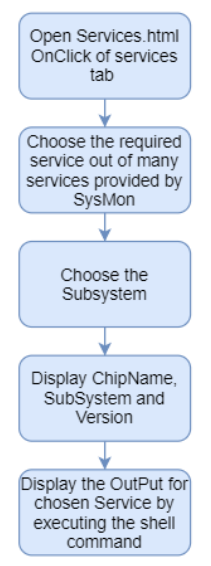

Fig 10: Flowchart of Services page

3.5 Report page: The Report tab will open when the user selects report from the NavBar. In a table, the name of the chipset, its subsystem, and its version will be displayed. On the UI, a table containing detailed SysMon Performance reports will be displayed.

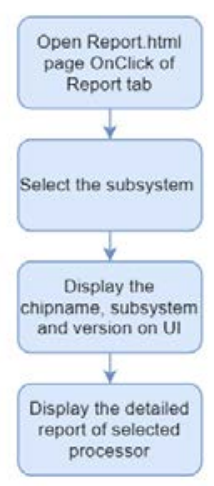

Fig 11 : Flowchart of Report page

3.6 User Guide page: When the user clicks the User Guide tab in the NavBar, SysMonGUI will navigate to the UserGuide tab. By selecting the relevant tab on the left side of the screen, the user guide of how to use SysMon is displayed in a text area. This is useful to learn and make the user aware of all the features available in SysMonApp GUI.

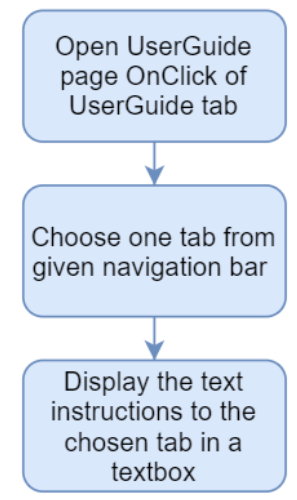

Fig 12: Flowchart of User Guide page

\section{Final GUI}

4.1 Home page:The home tab is the default tab that opens when the application is launched. The screen grab of the home tab is depicted in Figure 13. The various 
functionalities of the home tab are explained in detail below.

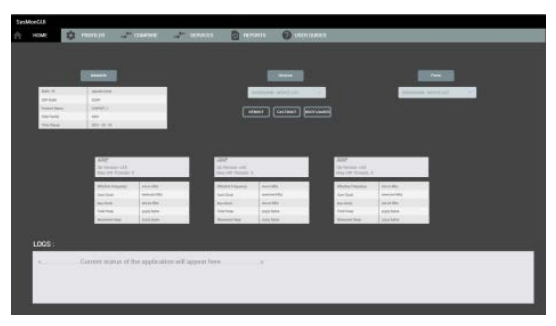

Fig 13: Home page

4.1.1 MetaInfo:The MetaInfo present on the top left of the screen depicts the metadata regarding the selected device. It gives the general information regarding the selected device, like it's build ID, DSP Build, Product Name, Chip Family and the Time Stamp. The MetaInfo is very helpful as it provides the key characteristics and identification details of the connected device. The connected device can sometimes be connected in a different geographic location, and the user who is accessing that connected device might be accessing the hardware from a remote location, and he/she might not have hardware or physical access to the device. By viewing the Metadata, a unique device can easily be identified.

4.1.2 Devices and Ports: There consists two dropdowns at the top of the screen; Devices and Ports. The device dropdown displays all the different connected devices that the SysMonGUI has access to. The connected device list is refreshed each time the app is launched. If there is no connected device, an alert message pops up on the screen that lets the user know that no devices are connected. The port dropdown lets the user to select the port. Once the device is selected from the dropdown, the metadata table and the processor parameters of that device is shown. Under the device dropdown list, there are three buttons, Reboot, Fastboot and Bootloader.

4.1.3 Parameters: Each device has different DSPs associated with it. Once the device is selected, the DSP parameters details become available. Parameters of different DSPs like Advanced Digital Signal Processor (ADSP), Cryptographical Digital Signal Processor (CDSP) and SDSP of that selected device is displayed. The different parameters that are shown are, the effective frequency, core clock speed, bus clock speed, total heap and measured heap. Along with the different parameters and the values, the Q6 version and the maximum number of hardware threads of the particular processor is also displayed, as shown in the figure.

4.1.4 Logs: This is the last section of the home tab on SysMonGUI. Here, the current status of the application appears here. This is only a developer feature, and helps to identify the commands being sent and received to the application. At any given point in time, the user can view the logs, which refresh in real time, to see the current status of the application

4.2 Profiler page: The profiler gives in depth analysis and metrics pertaining to the selected processor. Once the processor parameters are loaded in the home page, the user can click any one of the processor parameters, and the user will be directed to the profiler tab, with the clicked processor selected. The details of the device and the processor being evaluated is displayed on the top right of the screen, for the user's convenience. The screen grab of the profiler tab is depicted in Figure 14.

4.2.1 Clocks and Latency: On the top of the profiler screen, there are three dropdowns, where the user can specify the Core Clock Frequency, the Bus Clock Frequency and the Sleep Latency. This data helps in evaluating the processor and analysing its performance.

4.2.2 Metrics: On the top left of the screen, there are the different metrics that can be selected, on which the processor can be analysed. The different metrics that are available are All Metrics, Core Metrics, L1 Metrics, L2 Metrics, Bus Metrics, HMX Metrics, UDMA. 


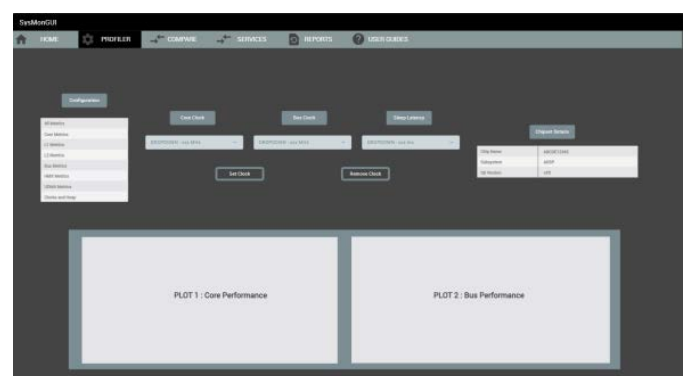

Fig 14: Profiler page

4.2.3 Performance Graphs:Once the Clocks and Latency is set, and the desired metric is selected, the performance graphs for the core performance and the Bus performance appears on the bottom half of the screen. These graphs are very helpful in analysing the performance characteristics of the processors. Since the clock speeds, latency can be controlled, the user has complete freedom to analyse the processor thoroughly.

4.3 Compare page: In the compare tab, users can compare different versions of the processors, and gain insights into the performance increase or decrease in successive versions of the processor. On the top of the screen, the selected device, and the processor is displayed. The screen grab of the compare tab is depicted in Figure 15.

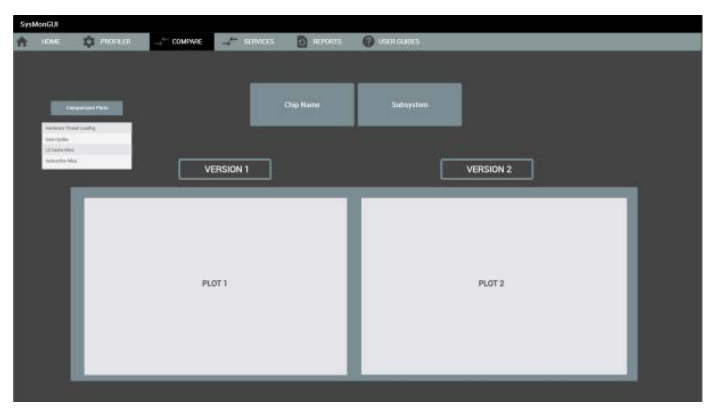

Fig 15: Compare page

4.3.1 Comparison parameters: The user can select the different comparison parameters on the top left of the screen. The different comparison parameters are Hardware Thread Loading, Core Cycles, L2 Cache Miss and Instruction Miss

4.3.2 Performance Graphs: Here, the performance of the two different versions of the processor is displayed side by side. This helps the user to compare different versions of the processor on the basis of a comparison metric.

4.4 Services page: SysMon offers a variety of services regarding the connected device and processor. On the right side, there are different services, like GetState, DCVS, Clocks, tinfo, SW Thread, Power Stats, that can be selected by the user. Once the user selects one of these services, the related output appears on the left side of the screen. The screen grab of the compare tab is depicted in Figure 16.

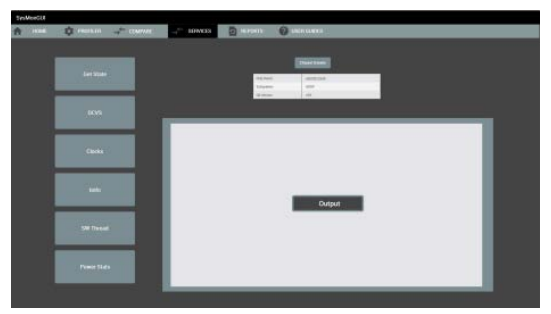

Fig 16: Services page

4.5 Report page: The reports tab gives detailed insight into the connected device and the processor. The overall Processor summary is displayed on the top of the screen. The selected device details and also the processor details is also displayed on the top-right of the screen. There are also additional reports that are displayed. Under Processor Summary, The Core Metrics, Bus Metrics, L1 Metrics reports are displayed. Under HVX Summary, Core Metrics, L2 Metrics, Stall metrics reports are displayed. The screen grab 
of the compare tab is depicted in Figure 17.

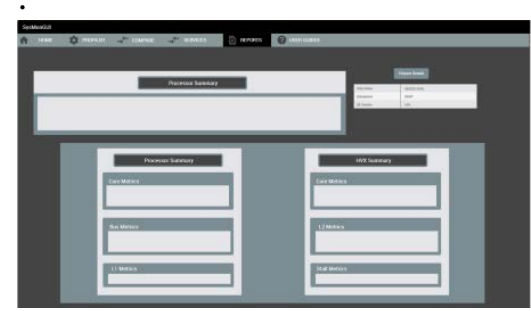

Fig 17 : Report page

4.6 User Guide page: The last and final tab of SysMonGUI is the User Guides Tab. This is a simple tab, with the main goal to inform users on how to use the application. It can be considered as a user manual for the application. On the right side of the screen there are six buttons, out of which, five buttons give the information regarding the five previous tabs discussed previously. The last button gives details and instructions about SysMon. When a button is present, the relevant details appear on the right side of the screen. The User Guides tab was created to make users aware all the features and functionalities of SysMonGUI. The screen grab of the compare tab is depicted in Figure 18.

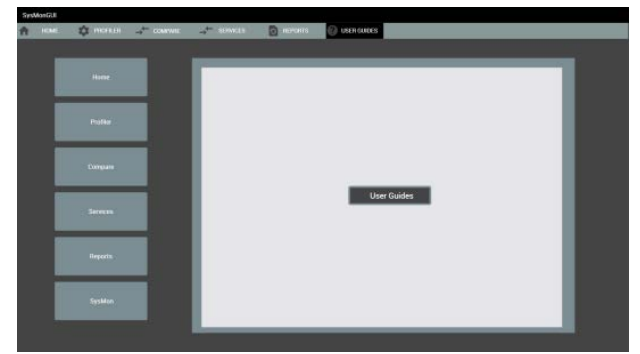

Fig 18: User Guide page

\section{Conclusion}

It was difficult for users to remember all of SysMonApp's parameters and arguments in order to use its functions. SysMonGUI brings together all of SysMon's functionality in one location, making it more user-friendly and simple to use. In the UI, there are six tabs for distinct SysMonApp functions. It also compares the performance of older and newer processor generations. All of the data is given in the form of graphs and tables to make it as simple as possible for the user to comprehend. Initially, the design of the GUI was decided. This helped to develop an understanding of how the GUI would look like. After the design was finalised, the app went through multiple rounds of revisions, to make it more userfriendly, and more functionally sound. After this, a mock up of the GUI was made. This was the protoyping phase, where the features and design elements that were the most suitable were added to the final version of the app.

\section{REFERENCES}

[1] G. Canfora, F. Mercaldo, C. A. Visaggio, M. D’Angelo, A. Furno, and C. Manganelli, "A case study of automating user experience-oriented performance testing on smartphones,” Mar. 2013, pp. 66-69, isbn: 978-1-4673-5961-0. doi: 10.1109/ICST.2013. 16

[2] Suraj Shahu Gaikwad and PRATIBHA ADKAR, "A Review Paper On Bootstrap Framework," ser. 10, vol. 2, IRE Journals, May 2019, pp. 349-351. [Online]. Available: https://www.irejournals.com/paper-details/1701173.

[3] F. S. Ocariza, K. Bajaj, K. Pattabiraman, and A. Mesbah, "A study of causes and consequences of client-side javascript bugs," IEEE Transactions on Software Engineering, vol. 43, no. 2, pp. 128-144, 2017. doi: 10.1109/TSE.2016.2586066. 
[4] P. Mussio, M. Finadri, P. Gentini, and F. Colombo, "A bootstrap approach to visual user-interface design and development," en, The Visual Computer, vol. 8, no. 2, pp. 75-93, Mar. 1992, issn: 0178-2789, 1432-8726. doi: 10.1007/BF01900550.[Online].Available:http://link.springer.com/10.1007/BF 01900550 (visited on 06/06/2021).

[5] H. Shah, "Node.js challenges in implementation," Global Journal of Computer Science and Technology, 2017, issn: 0975-4172. [Online]. Available: https://computerresearch. org/index.php/computer/article/view/1735.

[6] Pallavi Yadav and Paras Nath Barwal, "Designing Responsive Websites Using HTML And CSS," International Journal of Scientific \& Technology Research, vol. 3, no. 11, Nov. 2014, issn: 2277-8616. [Online]. Available: https://www.ijstr.org/paperreferences.php?ref=IJSTR-1114-10312.

[7] V. Avdiienko, K. Kuznetsov, I. Rommelfanger, A. Rau, A. Gorla, and A. Zeller, "Detecting behavior anomalies in graphical user interfaces," 2017 IEEE/ACM 39th International Conference on Software Engineering Companion (ICSE-C), pp. 201203, 2017.

[8] M. Welie, G. Veer, and A. Elins, "Patterns as tools for user interface design," Jun. 2000. doi: 10.1007/978-1-4471-0279-3_30 